Departures from therapeutic recommendations as defined by the Centers for Disease Control and Prevention, the American Thoracic Society, and the American College of Chest Physicians were considered errors in care. Management errors were detected in $28(80 \%)$ of the 35 patients, and there was an average of 3.93 errors per patient.

The most common errors included the addition of a single drug to a failing regime, the administration of an inadequate primary regime, failure to recognize primary or acquired drug resistance, failure to recognize and deal with patient noncompliance, and inappropriate preventive therapy.

The patients in whom management errors were made had a longer hospital stay and were treated with more total medications than those in whom no errors were detected. Seventeen surgical resections were performed for the management of tuberculosis. All were performed in patients who had management errors.

Acquired drug resistance occurred in 18 (86\%) of 21 patients subject to treatment errors and only one (14\%) of those without treatment error. Only two treatment failures occurred, both in the error group.

The authors concluded that the "pitfalls in the care of tuberculosis patients are multiple and common; these errors lead to the development of multidrugresistant isolates, the loss of efficacy of the most potent antituberculosis antibiotics, prolonged hospital stays and tremendous expenditures from the limited and precious healthcare budget."

FROM: Mahmoudi A, Iseman MD. Pitfalls in the care of patients with tuberculosis. Common errors and their association with the acquisition of drug resistance. JAMA 1993;270:65-68.

\section{CDC Revises Recommendations for Infection Control in Dentistry}

The CDC recently issued "Recommended Infection Control Practices for Dentistry, 1993." These guidelines update previously published CDC recommendations for infection control practices in dentistry to reflect new data, materials, technology, and equipment. These guidelines are intended to reduce risk of disease transmission in the dental environment from patient to dental healthcare worker (DHCW), from DHCW to patient, and from patient to patient. The recommendations include a call for routine use between patients of a heating process capable of sterilization (ie, steam under pressure [autoclaving], dry heat, or heat/chemical vapor) for all high-speed dental handpieces, low-speed handpiece components used intraorally, and reusable prophylaxis angles. Surface disinfection of these items by wiping or soaking in liquid chemical germicide is not an acceptable method for reprocessing.

FROM: Recommended Infection Control Practices for Dentistry, 1993. M M WR (Reports and Recommendations RR8) :42:1-12; May 28, 1993.

\section{CDC Finds a Dramatic Increase in Nosocomial Vancomycin-Resistant Enterococci}

As part of continual surveillance for antibiotic resistance among pathogens associated with nosocomial infections, a recent analysis of data from the Centers for Disease Control and Prevention's National Nosocomial Infections Surveillance (NNIS) System demonstrated a 20-fold increase from January 1, 1989, through March 31, 1993, in the percentage of enterococci associated with nosocomial infections resistant to vancomycin. Many of these strains are resistant to all available antimicrobial agents. The percentage of nosocomial enterococci resistant to vancomycin increased from $0.3 \%$ in 1989 to $7.9 \%$ in 1993 . Among patients in intensive care units (ICUs) with nosocomial infections, the percentage of enterococcal isolates resistant to vancomycin increased from $0.4 \%$ in 1989 to $13.6 \%$ in 1993 .

Vancomycin-resistant nosocomial enterococci have been reported from nine of 33 states with NNIS hospitals; the highest percentages were from NNIS hospitals in New York, Pennsylvania, and Maryland $(8.9 \%, 5.6 \%$, and $3.6 \%$ respectively).

Vancomycin resistance represents a serious challenge for physicians treating patients with bacterial infections, particularly because many hospitalacquired Enterococcus faecium strains also are resistant to beta-la\&m and aminoglycoside antibiotics. Treatment options for patients with nosocomial infections associated with vancomycin-resistant enterococci often are limited to unproven combinations of antimicrobials or experimental compounds.

Control measures for vancomycin-resistant enterococci include more consistent application of infection control precautions and control of indiscriminate vancomycin use.

FROM: Nosocomial enterococci resistant to vancomycin-United States, 1989-1993. M M WR 1993;42:597-599.

\section{New CDC Voice/FAX Service Available for Immunization Information}

The CDC and the National Immunization Program (formerly the CDC Division of Immunization, National Center for Prevention Services) have implemented a 24-hour, seven-days-a-week, automated FAX and voice 
system for easy access to immunization and travel information. The new service provides answers to questions most frequently asked by healthcare professionals and parents.

The FAX materials contain comprehensive and up-to-date information regarding immunizations, including those needed for foreign travel. The system's information ranges from brief summaries suitable for the general public to the detailed ACIP statements for each vaccine.

Much of the material is designed to educate the general public on immunizations that protect children against preventable diseases. Healthcare workers and practitioners may wish to direct interested patients to the system for reference.

To contact the Immunization Voice Information System, call (404) 332-4554 for information specific to immunizations, and select the topic of interest and method of delivery. Or dial direct to the CDC FAX Information Service, (404) 332-4565 and request the immunization directory. For access to the CDC Voice Information System, dial (404) 332-4555 for entry into the CDC-wide voice system, which contains other topics in addition to immunization.

\section{Severe Isoniazid-Associated Hepatitis Identified in New York}

After the identification of a patient in New York who underwent liver transplantation because of severe hepatitis that developed during the use of isoniazid (INH) preventive therapy for latent tuberculous infection, an inquiry into other liver transplant centers in New York identified additional patients who had hepatitis attributed to INH.

Ten patients from New York were identified as being evaluated for liver transplantation because of severe acute hepatitis attributed to INH from January 1991 through May 1993. Of the 10 patients, one received both INH and rifampin. Another had received INH and discontinued its use one month before the onset of hepatitis symptoms because the patient had been exposed to tuberculosis resistant to INH and rifampin. Eight patients were taking INH alone (as therapy to prevent TB) at the onset of the hepatitis. The eight were aged 5 to 68 years; three were aged a20 years. Six were female. Because of the severity of illness, five of the eight patients received a liver transplant; one of these patients died after transplantation. The other three patients died while awaiting a donor liver.

The duration of INH use before the onset of hepatitis symptoms was either known or could be estimated for seven patients and ranged from 21 to 142 days; seven patients continued to take INH for at least 10 days after onset of symptoms. Initial symptoms of hepatitis included fatigue in five patients, nausea in five, abdominal pain in five, and anorexia in four. All patients had jaundice when they sought medical attention.

Previous reports have described cases of severe or fatal INH-associated hepatitis. One study estimated the risk for hepatitis as 20.7 per 1,000 persons with $4.6 \%$ of the cases being fatal. However, in this report neither the number of persons in New York who receive INH preventive therapy each year nor the number who have INH-associated hepatitis are known. Thus, it is unclear whether the number of patients in this report represents an increase in severe or lifethreatening INH-associated hepatitis or an improvement in the detection of the problem.

FROM: Severe isoniazid-associated hepatitis-New York, 1991-1993. MMWR 1993;42:545-547.

\section{Long-Term Acyclovir for Genital Herpes Is Safe and Effective}

The Acyclovir Study Group now has reported results through the fifth year of their ongoing long-term clinical trial.

After the first year of the study, all patients were offered open-label acyclovir in a dose of $400 \mathrm{mg}$ twice daily. Three hundred eighty-nine patients completed their fifth year of suppressive therapy and $25 \%$ of the patients had no recurrence for the entire five years. During each quarter of the fifth year, $86 \%$ to $90 \%$ of patients had no recurrence. The mean annual number of recurrences declined from 1.7 during the first year of the study to 0.8 during the fifth year of suppressive therapy. A small number of patients $(3.3 \%)$ needed a dose of $800 \mathrm{mg}$ acyclovir to suppress the outbreaks.

There were no significant changes in white blood cell counts, creatinine, or liver function tests in patients during the fifth year of treatment, and no cumulative toxicity was observed over time.

FROM: Goldberg LH, et al. Long-term suppression of recurrent genital herpes with acyclovir. A rch Dermatol 1993;129:582-587.

\section{MEDWatch Launched to Improve Adverse Events Reporting}

The U.S. Food and Drug Administration (FDA) recently announced a program to improve the safety of drugs, biologics, medical devices, infant formulas, dietary supplements, medical foods, and other regulated products by encouraging health professionals voluntarily to report any serious adverse events and product defects.

The new program, called MEDWatch, is a comprehensive approach to FDA's postmarketing surveillance, and is aimed at improving health professionals' involvement by focusing on their reporting.

"Physicians, nurses, and others who care for patients are the first to know when a drug or medical 